\title{
Prevalence of small cerebral bleeds in patients with progressive supranuclear palsy: a neuropathological study with 7.0-Tesla magnetic resonance imaging correlates
}

\author{
Jacques De Reuck ${ }^{1,2}$, Dominique Caparros-Lefebvre ${ }^{3}$, Vincent Deramecourt ${ }^{1,2,4,5}$, Luc Defebvre ${ }^{1,4,6}$, Florent Auger ${ }^{1,2}$, \\ Nicolas Durieux ${ }^{1,2}$, Regis Bordet ${ }^{1,2}$, Florence Pasquier ${ }^{1,2,4}$, Claude-Alain Maurage ${ }^{1,4,5}$ \\ ${ }^{1}$ Université Lille Nord de France, F-59000 Lille, ${ }^{2}$ UDSL, EA 1046, F-59000 Lille, ${ }^{3}$ Centre Hospitalier, F-59150 Wattrelos, ${ }^{4} \mathrm{CHULille}$, \\ F-59000 Lille, ${ }^{5}$ INSERM U837, F-59000 Lille, ${ }^{6}$ UDSL, EA 4559, F-59000 Lille, France
}

\begin{abstract}
Introduction: Progressive supranuclear palsy (PSP) is a degenerative disease affecting mainly the brain stem, basal ganglia and cerebellum. Associated cerebrovascular lesions, mainly small cerebral bleeds, are frequently observed in some neurodegenerative diseases such as Alzheimer dementia and rare in others such as frontotemporal lobar degeneration. The present post-mortem study investigates the prevalence and distribution of small cerebral bleeds in PSP brains.

Material and methods: Nineteen brains of PSP patients were compared to 12 age-matched controls. The prevalence and distribution of mini-bleeds were investigated on a coronal section of a cerebral hemisphere at the level of the mamillary bodies and on a horizontal section through the pons and cerebellum. In addition, out of these series T2*-weighted gradient-echo 7.0-Tesla magnetic resonance imaging (MRI) of 3 coronal sections of a cerebral hemisphere and of a brain stem and cerebellum was performed in 14 PSP and 11 control brains.

Results: Although the total number of mini-bleeds was the same on neuropathological examination of both groups, they prevailed around the dentate nucleus of the cerebellum $(p=0.05)$ and in the tegmentum pontis $(p=0.05)$ of the PSP brains. On MRI the small bleeds were also more frequent around the dentate nucleus of the cerebellum $(p=0.02)$ and in the pons $(p=0.04)$ of PSP brains.

Discussion: In PSP brains, mini-bleeds only prevail in the regions affected by the neurodegenerative process, similarly to what happens in frontotemporal lobar degeneration. They should be considered as the result of increased angiogenesis and microglial activation, leading to associated disturbances of the blood-brain barrier in the most affected regions of PSP. They are not indicative of cerebrovascular disease.
\end{abstract}

Key words: progressive supranuclear palsy, neuropathology, T2*-weighted gradient-echo 7.0-T MRI, tauopathies, small cerebral bleeds, cerebellum, pons. 


\section{Introduction}

Progressive supranuclear palsy (PSP) was first described in 1964 by Steele, Richardson and Olszewski as a heterogeneous degeneration involving the brain stem, basal ganglia and cerebellum with clinically a vertical gaze and pseudobulbar palsy, nuchal dystonia and dementia [21]. Progressive supranuclear palsy is now increasingly recognized, with an average prevalence of 3.5 per 100000 [19]. It belongs to the family of tauopathies with the classical picture, consisting of a symmetrical akinetic-rigid syndrome, vertical supranuclear gaze palsy, frontal deficits, prominent postural instability and falls, now referred to as Richardson's syndrome (PSP-RS). However, several variants in the clinical presentation complicate the early differential diagnosis [20]. It now includes a progressive supranuclear palsy-parkinsonism (PSP-P) form and one with pure akinesia and gait freezing (PAGF) [22].

Vascular factors have sometimes been implicated, leading to the description of a vascular progressive supranuclear palsy syndrome [15]. A pre-symptomatic history of arterial hypertension as a major feature in the diagnosis of PSP remains controversial $[3,13]$.

Although the co-existence of argyrophilic grains, corticobasal degeneration, Alzheimer features and Lewy bodies was rather frequent in a large post-mortem series of PSP patients, the severity of cerebrovascular pathology was found to be too limited to explain any clinical symptomatology [17]. These findings are in contrast to the high incidence of small bleeds observed in brains of patients with Alzheimer dementia (AD) and Lewy body dementia (LBD) $[4,5,9]$.

The present neuropathological and post-mortem 7.0-Tesla magnetic resonance imaging (MRI) study investigates the prevalence and the distribution of small cerebral bleeds and other cerebrovascular lesions in patients with PSP.

\section{Material and methods}

\section{Progressive supranuclear palsy and control population}

From January 2000 until February 2013, 212 consecutive patients with a clinical history of a neurodegenerative disease came to autopsy: in 19 (9\%) of them the neuropathological diagnosis of PSP was made.

During the same period, 12 post-mortem brains of age-matched controls were obtained. The controls consisted of brains of elderly patients who died from an illness not related to a cerebral disease.

All patients had well-documented clinical files including a prior history of vascular risk factors and of the use of antithrombotic agents.

\section{Neuropathological analyses}

Post-mortem brains were obtained by informed consent of the patients or from the nearest family, who allowed an autopsy for a post-mortem diagnosis and the use of brain samples for scientific purposes. The brain tissue samples were first used for diagnosis and afterward integrated in the Lille Neuro-Bank, dependent on the University of Lille and co-federated by the "Centre des Resources Biologiques", acting as the institutional review board. The neuropathological evaluation was performed blinded to history and clinical data.

\section{Neurodegenerative lesions}

After 3-week fixation in formalin, many samples were taken according to a standard protocol [4]. Slides from paraffin-embedded sections were immuno-stained for tau protein, $\beta$-amyloid, $\alpha$-synuclein, prion protein, TDP-43 and ubiquitin.

The post-mortem diagnosis of PSP was made according to the NINDs neuropathological criteria for Steele-Richardson-Olszewski syndrome (progressive supranuclear palsy) [14]. Additional AD features were staged according to the classification of Braak and Braak [1].

\section{Cerebrovascular lesions}

The prevalence and severity of cerebrovascular lesions were compared between the brains of PSP patients and controls. In addition, a semi-quantitative evaluation of small microscopic lesions was performed on a whole coronal section of a cerebral hemisphere, at the level of the mamillary bodies, and a horizontal section through the mid-pons and cerebellar hemispheres, after staining with hematoxylin-eosin, Luxol Fast Blue and Perl's Prussian Blue. The considered cerebrovascular lesions were bleeds, infarcts, lacunes and white matter changes.

A semi-quantitative scale, with ranking (R) 0 to 3 , was used to evaluate the severity of the white matter changes and the frequency of micro-infarcts and of micro- and mini-bleeds. The white matter changes were restricted to the periventricular regions (R1), 
scattered in the centrum semiovale (R2) or forming confluent lesions (R3) of myelin and axonal loss. For the micro-infarcts, micro- and mini-bleeds R1 corresponded to 1 to 2, R2 up to 5 and R3 to more than 5 lesions per region [4].

\section{Magnetic resonance imaging examination}

Out of the neuropathological series, 14 PSP and 11 control brains were available for MRI examination. On a 7.0-Tesla MRI Bruker BioSpec SA (Ettlingen, Germany) three MRI sequences were used: a positioning sequence, a $\mathrm{T} 2$ sequence and a gradient echo $\mathrm{T}^{*}$ sequence. Three coronal sections of a formalinfixed cerebral hemisphere and one section of brain stem and cerebellar hemisphere, placed in a plastic box filled with physiological serum, were examined according to the previously described method [6]. The mean number of cortical small bleeds detected on each examined section was compared between the PSP and control brains. The majority of the brain stem sections consisted of a horizontal section through the pons. Only in one PSP and one control brain was a sagittal section of the whole brain stem available.

\section{Statistical analyses}

The statistical analysis compared the items of the PSP with the control group. Univariate comparisons of unpaired groups were done with Fisher's exact test for categorical data. The non-parametric Mann-Whitney $U$-test was used to compare continuous variables. The significance level was set at 0.05 , two-tailed.

\section{Results}

The demographic and main clinical data of the 19 patients with proven PSP are labeled in Table I.

Table I. Demographic, clinical and associated neuropathological features of the 19 patients with progressive supranuclear palsy

\begin{tabular}{|c|c|c|c|c|}
\hline $\begin{array}{l}\text { Age at disease onset } \\
\text { (years) }\end{array}$ & Gender & Clinical syndrome & Age at death (years) & Associated pathology \\
\hline 50 & Female & Richardson & 55 & \\
\hline 50 & Male & Richardson & 57 & \\
\hline 51 & Male & Richardson & 57 & \\
\hline 56 & Female & Parkinson & 65 & \\
\hline 59 & Female & Richardson & 72 & \\
\hline 59 & Female & Parkinson & 74 & \\
\hline 61 & Male & Parkinson & 68 & Alzheimer II \\
\hline 66 & Female & Richardson & 69 & \\
\hline 67 & Female & Richardson & 82 & \\
\hline 68 & Female & Richardson & 77 & \\
\hline 71 & Female & Parkinson & 77 & \\
\hline 71 & Male & Parkinson & 83 & \\
\hline 72 & Female & Parkinson & 77 & \\
\hline 72 & Male & Richardson & 82 & \\
\hline 73 & Male & Parkinson & 75 & Alzheimer III \\
\hline 73 & Female & Parkinson & 87 & \\
\hline 75 & Female & Parkinson & 79 & \\
\hline 78 & Male & Richardson & 87 & \\
\hline 79 & Male & Richardson & 83 & Alzheimer II \\
\hline
\end{tabular}


Table II. Comparison of the number (percentage) of patients with vascular risk factors and the use of antithrombotic medication among the group with progressive supranuclear palsy (PSP) and among controls

\begin{tabular}{|lccc|}
\hline Items & $\begin{array}{c}\text { PSP } \\
(n=19)\end{array}$ & $\begin{array}{c}\text { Controls } \\
(n=12)\end{array}$ & $p$ value \\
\hline Arterial hypertension & $7(37)$ & $3(25)$ & 0.70 \\
\hline Diabetes & $1(5)$ & $1(8)$ & 1.0 \\
\hline Hypercholesterolemia & $1(5)$ & $2(17)$ & 0.54 \\
\hline Smoking & $1(5)$ & $1(8)$ & 1.0 \\
\hline $\begin{array}{l}\text { Antithrombotic } \\
\text { treatment }\end{array}$ & $6(32)$ & $3(25)$ & 1.0 \\
\hline
\end{tabular}

The median age at onset of the PSP was 67 (interquartile range [IQR]: 56-73) years). Ten patients presented clinically as a PSP-RS and 9 as a PSP-P. The average disease duration was 8.5 (SD 4.3) years. The median age of the patients at death was 75 (IQR: 68-82) years. This was not statistically different from the 70 (IQR 65-77) years in the controls. Gender distribution was also similar, with $42 \%$ males in the former and $75 \%$ in the latter group.

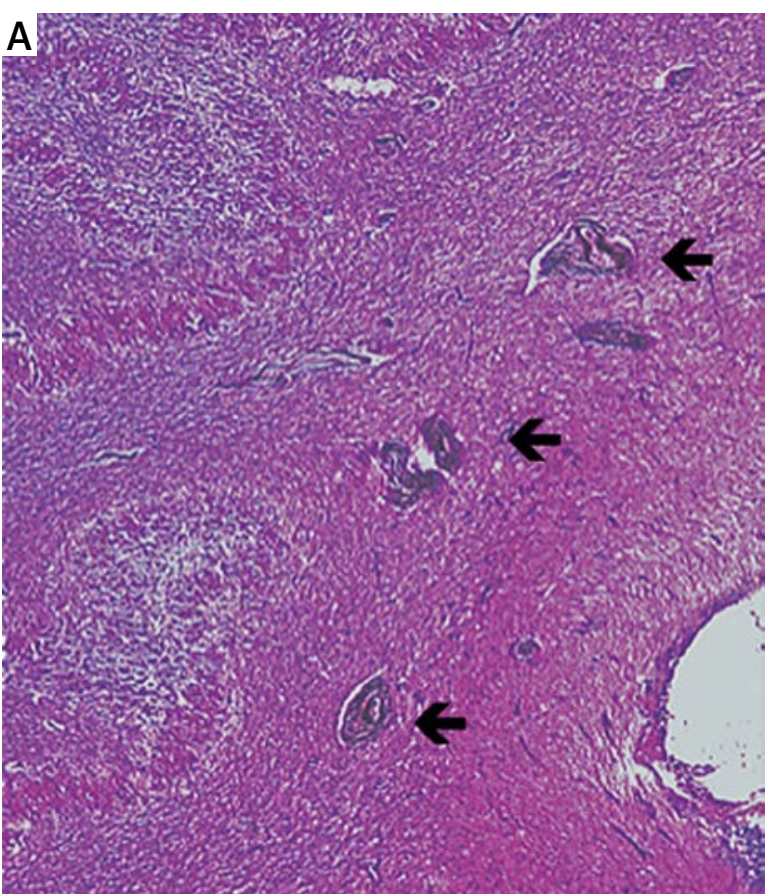

The vascular risk factors of both groups were rare and not significantly different between the groups. Antithrombotic agents had been used by $32 \%$ of the PSP patients and by $25 \%$ of the controls (Table II).

The global severity of mini-bleeds was low in the PSP brains and comparable to the controls on neuropathological examination. Only mini-bleeds in the cerebellum $(p=0.05)$ and in the pons $(p=0.05)$ were significantly more frequent in PSP brains. In the cerebellum the mini-bleeds were exclusively found around the dentate nucleus, while in the pons they predominated in the tegmentum (Fig. 1A-B). The incidence of other cerebrovascular lesions was low in the PSP as well as in the control brains (Table III).

Table IV shows the mean values of hypo-intensity signals, representing small bleeds, in the cerebral cortex of the 3 coronal slides of a cerebral hemisphere, the hippocampus, pons and cerebellum on T2*-weighted gradient-echo MRI. Only around the dentate nucleus of the cerebellum and in the tegmentum pontis of the PSP brains, significantly more small bleeds were observed compared to the controls $(p<0.5)$ (Fig. 2A-B). One sagittal sample of a whole PSP brainstem could be compared to a normal control. In addition to the global atrophy and the

Fig. 1. Histological sections of cerebellum and pons, stained with hematoxylin-eosin, of a patient with progressive supranuclear palsy. A) Presence of three small perivascular bleeds (arrows) near the dentate nucleus of the cerebellum. B) Small perivascular bleed in the dorsal part of the pons.

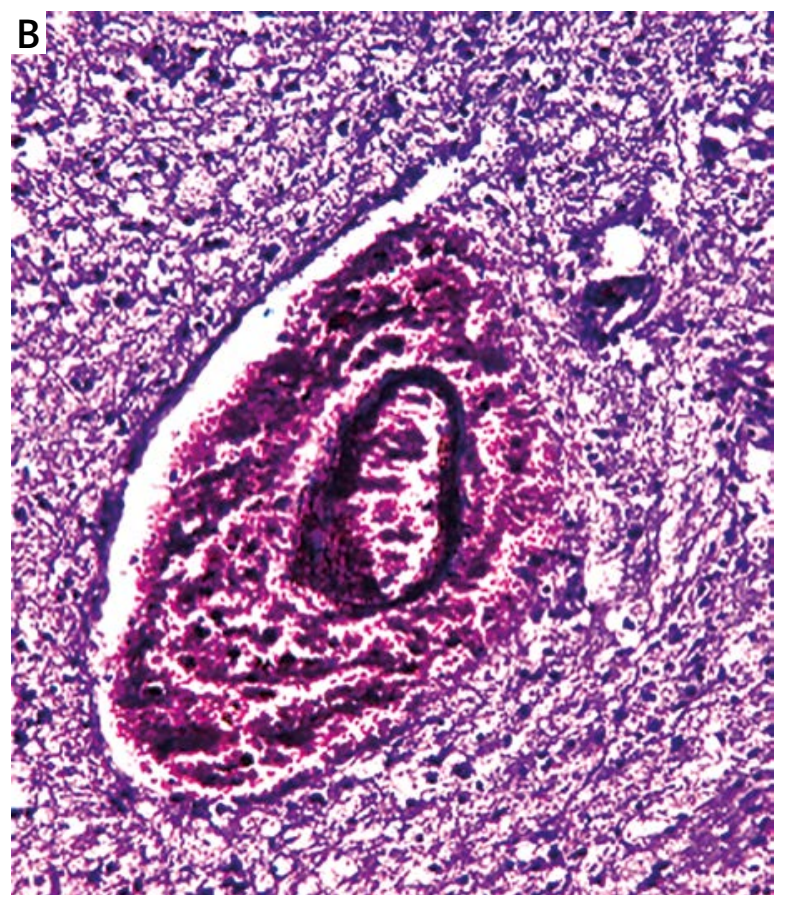


Table III. Neuropathological comparison of the mean ranking scores (standard deviations) of the severity of cerebrovascular lesions among patients with progressive supranuclear palsy (PSP) and among controls

\begin{tabular}{|lccc|}
\hline Items & $\begin{array}{c}\text { PSP } \\
(n=19)\end{array}$ & $\begin{array}{c}\text { Controls } \\
(n=12)\end{array}$ & $p$ value \\
\hline White matter changes & $0.6(1.0)$ & $0.3(0.9)$ & 0.33 \\
\hline Amyloid angiopathy & $0.1(0.2)$ & $0.0(0.0)$ & 0.82 \\
\hline Lacunar infarcts & $0.1(0.2)$ & $0.1(0.3)$ & 0.91 \\
\hline Territorial infarcts & $0.2(0.5)$ & $0.0(0.0)$ & 0.62 \\
\hline Cortical micro-infarcts & $0.2(0.5)$ & $0.0(0.0)$ & 0.43 \\
\hline Hematomas & $0.1(0.2)$ & $0.0(0.0)$ & 0.82 \\
\hline Micro-bleeds & $0.0(0.0)$ & $0.0(0.0)$ & 1.0 \\
\hline Mini-bleeds & $1.3(1.0)$ & $0.5(0.5)$ & 0.07 \\
\hline Total & $0.5(0.9)$ & $0.4(0.5)$ & 1.0 \\
\hline \begin{tabular}{l} 
Cortico-subcortical \\
\hline White matter
\end{tabular} & $0.8(0.8)$ & $0.5(0.7)$ & 0.38 \\
\hline \begin{tabular}{l} 
Striatum \\
\hline Thalamus
\end{tabular} & $0.4(0.6)$ & $0.2(0.4)$ & 0.61 \\
\hline $\begin{array}{l}\text { Cons } \\
\text { Cerebellum }\end{array}$ & $0.1(0.2)$ & $0.1(0.3)$ & 0.89 \\
\hline
\end{tabular}

A

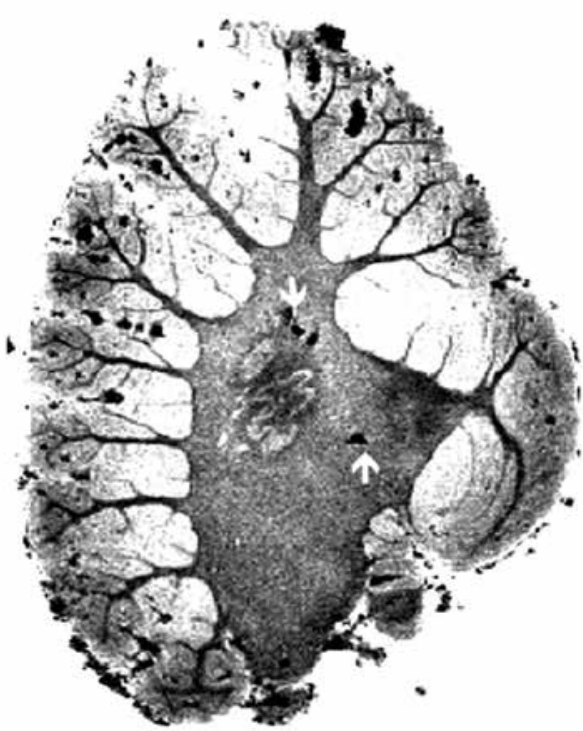

Table IV. Comparison of the mean number (standard deviation) of small bleeds on $\mathrm{T}^{*}$-weighted gradient-echo MRI in post-mortem brains among patients with progressive supranuclear palsy (PSP) and among controls

\begin{tabular}{|lccc|}
\hline Items & $\begin{array}{c}\text { PSP } \\
(n=14)\end{array}$ & $\begin{array}{c}\text { Controls } \\
(n=11)\end{array}$ & $p$ value \\
\hline Frontal cerebral cortex & $2.5(2.3)$ & $3.2(2.5)$ & 0.40 \\
\hline Central cerebral cortex & $2.3(2.0)$ & $1.6(1.6)$ & 0.47 \\
\hline Occipital cerebral cortex & $5.2(4.0)$ & $3.2(2.4)$ & 0.34 \\
\hline Hippocampus & $0.1(0.4)$ & $0.5(1.0)$ & 0.57 \\
\hline Pons & $1.8(1.4)$ & $0.3(0.5)$ & 0.04 \\
\hline Cerebellum & $3.1(2.3)$ & $0.6(0.9)$ & 0.02 \\
\hline
\end{tabular}

loss of hypo-intensity in the substantia nigra and red nucleus, small bleeds were observed in the dorsal part of the whole brain stem (Fig. 3).

\section{Discussion}

No overall increase of small cerebral bleeds is observed in PSP brains on neuropathological examination or on MRI. This is similar to what happens in frontotemporal lobar degeneration, which in most cases also belongs to the family of tauopathies [7]

B

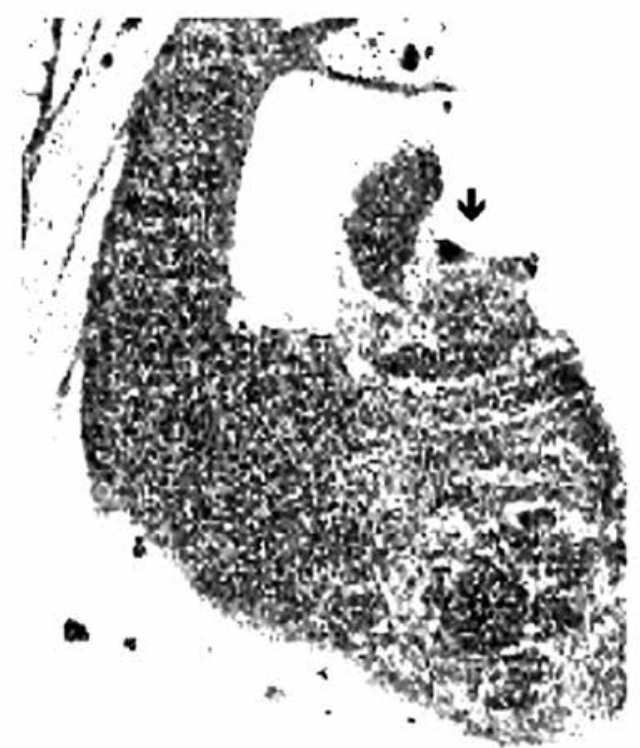

Fig. 2. T2*-weighted gradient-echo 7.0-T magnetic resonance imaging of a horizontal hemi-section of a cerebellar hemisphere and pons in progressive supranuclear palsy. A) Presence of small bleeds around the dentate nucleus (arrows). B) One small subependymal bleed in the pons (arrow). 

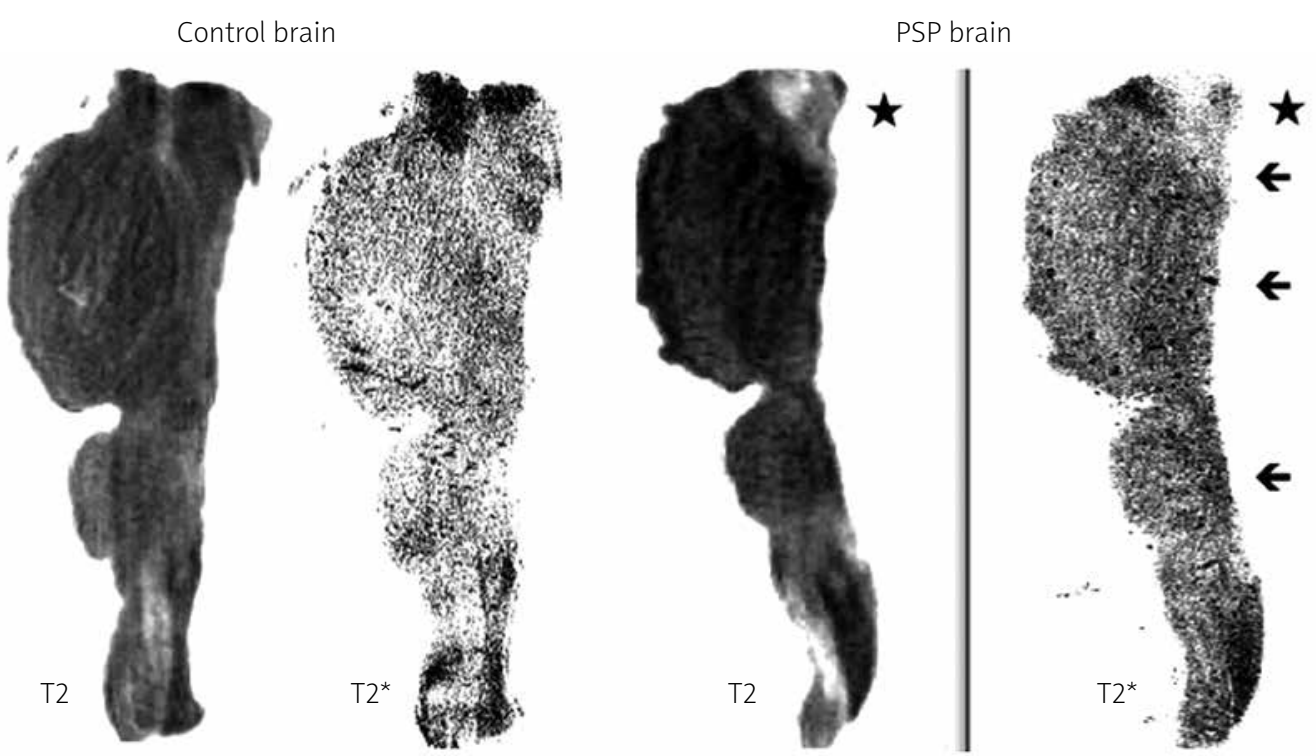

Fig. 3. T2 and T2-weighted gradient-echo 7.0-T magnetic resonance imaging of a sagittal section through the whole brain stem of a control and of a patient with progressive supranuclear palsy (PSP). Note the global atrophy of the PSP brain stem as well as the decreased iron content in the substantia nigra and red nucleus (asterisks). Small bleeds are more prominent in the dorsal part of the whole brain stem in the PSP compared to the control (arrows).

and is in contrast to the overall prevalence of small bleeds in $A D$ and in the cerebral cortex of $\operatorname{LBD}[4,5,9]$. However, compared to age-matched controls, an increased number of mini-bleeds is observed around the dentate nucleus of the cerebellum and in the tegmentum pontis of PSP brains.

Ponto-cerebellar micro-bleeds are frequently observed in patients with cerebral micro-angiopathy due to arterial hypertension [12] and in those using antithrombotic agents [2]. These bleeds are mainly observed around the dentate nucleus of the cerebellum, similar to our cases, and in the central portion of the pons [18] instead of in the tegmentum as in the PSP brains. Our patients with PSP have a low incidence of vascular risk factors, similar to the controls.

Our 7.0-Tesla MRI study confirms the neuropathological findings of an increased incidence of small bleeds in the pons and cerebellum. In the sample covering the whole brain stem, one can suspect that the small bleeds extend over the whole length of the brain stem.

The selective presence of mini-bleeds must be linked to the neurodegenerative process of the PSP itself. The clinical and the neuropathological findings in PSP are heterogeneous and can affect the cerebellum to various degrees $[16,22]$.
The findings in PSP share similarity with those observed in our post-mortem 7.0-Tesla MRI study of frontotemporal lobar degeneration, in which minibleeds predominated in the frontal cortex, where the major neurodegenerative changes occur [8].

There is now evidence for increased angiogenesis and microglial activation in the neuropathologically most affected regions of PSP. Such angiogenic vessels could contribute to neuroinflammation and lead to disruption of the blood-brain barrier [11]. Also in Alzheimer's disease ongoing angiogenesis is mainly observed in the hippocampus and is correlated with the presence of neurofibrillary tangles [10].

In any case, the presence of these mini-bleeds in the pons and cerebellum are not to be considered as evidence of cerebrovascular involvement in the pathogenesis of PSP.

\section{Disclosure}

Authors report no conflict of interest.

\section{References}

1. Braak H, Braak E. Evolution of neuronal changes in the course of Alzheimer's disease. J Neural Transm 1998; 53 (Suppl): 127-140. 
2. Chang YY, Liu JS, Lai SL, Wu HU, Lan MY. Cerebellar hemorrhage provoked by combined use of natokinase and aspirin in a patient with cerebral microbleeds. Inter Med 2008; 47: 467-469.

3. Colosimo C, Osaki Y, Vanacore N, Lees AJ. Lack of association between progressive supranuclear palsy and arterial hypertension: a clinicopathological study. Mov Dis 2003; 18: 694-697.

4. De Reuck J, Deramecourt V, Cordonnier C, Leys D, Pasquier F, Maurage CA. Prevalence of small cerebral bleeds in patients with a neurodegenerative dementia: a neuropathological study. J Neurol Sci 2011; 300: 63-66.

5. De Reuck J, Deramecourt V, Cordonnier C, Leys D, Maurage CA, Pasquier $F$. The impact of cerebral amyloid angiopathy on the occurrence of cerebrovascular lesions in demented patients with Alzheimer features: a neuropathological study. Eur J Neurol 2011; 18: 313-318.

6. De Reuck J, Auger F, Cordonnier C, et al. Comparison of 7.0$\mathrm{T} T 2^{*}$-magnetic resonance imaging of cerebral bleeds in post-mortem brain sections of Alzheimer patients with their neuropathological correlates. Cerebrovasc Dis 2011; 31: 511-517.

7. De Reuck J, Deramecourt V, Cordonnier C, Leys D, Pasquier F, Maurage CA. Cerebrovascular lesions in patients with frontotemporal lobar degeneration: a neuropathological study. Neurodegenerative Dis 2012; 9: 170-175.

8. De Reuck J, Deramecourt V, Cordonnier C, et al. Detection of microbleeds in post-mortem brains of patients with frontotemporal lobar degeneration: A 7.0 tesla magnetic resonance imaging study with neuropathological correlates. Eur J Neurol 2012; 19: 1355-1360.

9. De Reuck J, Deramecourt V, Cordonnier C, Leys D, Pasquier F, Maurage CA. Prevalence of cerebrovascular lesions in patients with Lewy body dementia: A neuropathological study. Clin Neurol Neurosurg 2013; 115: 1094-1097.

10. Desai BS, Schneider JA, Li JL, Carvey PM, Hendey B. Evidence of angiogenetic vessels in Alzheimer's disease. J Neural Transm 2009; 116: 587-579.

11. Desai Bradaric B, Patel A, Schneider JA, Carvey PM, Hendey B. Evidence of angiogenesis in Parkinson's disease, incidental Lewy body disease, and progressive supranuclear palsy. J Neural Transm 2012; 119: 59-71.

12. Fazekas F, Kleinert R, Roob G, et al. Histopathologic analysis of foci of signal loss on gradient-echo T2*-weighted MR images in patients with spontaneous intracerebral hemorrhage: evidence of microangiopathy-related microbleeds. Am J Neuroradiol 1999; 20: 637-642.

13. Ghika J, Bogousslavsky J. Presymptomatic hypertension is a major feature in the diagnosis of progressive supranuclear palsy. Arch Neurol 1997; 54: 1104-1108.

14. Hauw JJ, Daniel SE, Dickson D, et al. Preliminary NINDS neuropathological criteria for Steele-Richardson-Olszewski syndrome (progressive supranuclear palsy); Neurology 1994; 44: 2015 2019.

15. Joseph KA, Ishizawa T, Tsuboi Y, Cookson N, Dickson DW. A clinico-pathological study of vascular progressive supranuclear palsy. A multi-infarct disorder presenting as progressive supranuclear palsy. Arch Neurol 2002; 59: 1597-1601.

16. Kanzawa M, Shimohata T, Toyoshima Y, et al. Cerebellar involvement in progressive supranuclear palsy: A clinicopathological study. Mov Dis 2009; 24: 1312-1318.
17. Keith-Rokosh J, Ang LC. Progressive supranuclear palsy: a review of co-existing neurodegeneration. Can J Neurol Sci 2008; 35 : 602-608.

18. Lee SH, Kwon SJ, Kim KS, Yoon BW, Roh JK. Topographic distribution of pontocerebellar microbleeds. Am J Neuroradiol 2004; 25: 1337-1341.

19. Nath U, Ben-Shlomo Y, Thompson RG, et al. The prevalence of progressive supranuclear palsy (Steele-Richardson-Olszewski) in the UK. Brain 2001; 124: 1438-1449.

20. Stamelou M, de Silva R, Arias-Carrion O, et al. Rational therapeutic approaches to progressive supranuclear palsy. Brain 2010; 133: 1578-1590.

21. Steele JC, Richardson C, Olszewski J. Progressive supranuclear palsy. A heterogeneous degeneration involving the brain stem, basal ganglia and cerebellum with vertical gaze and pseudobulbar palsy, nuchal dystonia and dementia. Arch Neurol 1964; 10: 333-359.

22. Williams DR, Holton JL, Strand C, et al. Pathological tau burden and distribution distinguishes progressive supranuclear palsy-parkinsonism from Richardson's syndrome. Brain 2007; 130: 1566-1576. 